\title{
Shape-Induced Frustration of Hexagonal Order in Polyhedral Colloids
}

\author{
Roel P. A. Dullens, ${ }^{1, *}$ Maurice C. D. Mourad, ${ }^{1}$ Dirk G. A. L. Aarts, ${ }^{1}$ Jacob P. Hoogenboom, ${ }^{2}$ and Willem K. Kegel ${ }^{1}$ \\ ${ }^{1}$ Van't Hoff Laboratory for Physical and Colloid Chemistry, Debye Institute, Utrecht University, \\ Padualaan 8, 3584 CH Utrecht, The Netherlands \\ ${ }^{2}$ Soft Condensed Matter, Debye Institute, Utrecht University, Princetonplein 5, 3584 CC Utrecht, The Netherlands
}

(Received 17 January 2005; published 18 January 2006)

\begin{abstract}
The effect of a nonspherical particle shape and shape polydispersity on the structure of densely packed hard colloidal particles was studied in real space by confocal microscopy. We show that the first layer at the wall of concentrated size-monodisperse but shape-polydisperse polyhedral colloids exhibits significant deviations from a hexagonal lattice. These deviations are identified as bond-orientational fluctuations which lead to percolating "mismatch lines." While the shape-induced geometrical frustration of the hexagonal symmetry suppresses translational order, bond-orientational order is clearly retained, indicating a hexaticlike structure of the polyhedral colloids.
\end{abstract}

DOI: 10.1103/PhysRevLett.96.028304

Polydispersity is an intrinsic property of colloidal systems. For hard spheres it is well known that size polydispersity has a tremendous effect on the structure and dynamics [1-6]. However, the effect of the particle shape is a more general issue, as molecular systems are "monodisperse" in size but usually not spherical. The impact of shape is, for example, demonstrated in simulations that show the existence of a rotator-phase in hard hexamers, pentamers, and pentagons [7-10], being absent in hard spheres. The influence of shape polydispersity on crystallization has hardly been addressed, despite the fact that relevant systems like nanoparticles [11] and granular matter [12] often exhibit a distribution of particle shapes. Here, we use a colloidal model system to study the influence of only the particle shape on the structure of densely packed particles. In particular, the particles represent hard spheres that are monodisperse in size, but have a small random perturbation in shape.

The model colloids are crosslinked and fluorescently labeled polymethylmethacrylate (PMMA) particles. Their preparation is described in Ref. [13]. As shown in the inset in Fig. 1(a), these particles clearly have a nonspherical, polyhedral shape, which was also observed using electron microscopy. Note, that "polyhedral" refers to the twodimensional (2D) cross section through the center of the three-dimensional particle. The particle shape can be characterized by a shape factor $\mathrm{SF}=4 \pi A_{p} / P_{p}^{2}$, with $A_{p}$ the projected particle area and $P_{p}$ the projected perimeter. The average SF for the polyhedrals is $0.76 \pm 0.09$ compared to 1 for a perfect sphere. The random character of the nonsphericity is reflected by the polydispersity in shape of $11.8 \%$. Comparison to regular polygons reveals that the polyhedrals are on average best described by pentagons. Remarkably, their size polydispersity is comparable to that of rather monodisperse colloidal spheres. Therefore, these polyhedrals provide an excellent model system to investigate the effect of shape and shape polydispersity. In our experiment, we compare the structure of the first layer at the wall of densely packed polyhedrals to one formed by
PACS numbers: 82.70.Dd, 64.60.Cn, 64.70. $-\mathrm{p}$

spherical and size-monodisperse reference PMMA particles [inset Fig. 1(b)] [14]. The diameters $d$ and shape factors are respectively $d=2.23 \pm 0.09 \mu \mathrm{m}, \mathrm{SF}=$ $0.76 \pm 0.09$ (polyhedrals) and $d=2.33 \pm 0.07 \mu \mathrm{m}, \mathrm{SF}=$ $0.96 \pm 0.02$ (spheres). Both systems were dispersed in a mixture of cis-decalin, tetralin, and carbontetrachloride, which matches the refractive index and almost the mass density of the particles [15]. In this solvent, the PMMA particles interact via a hard-sphere-like potential [15]. For both systems a sample with a volume fraction $\phi \approx 0.40$ was prepared by diluting a random close packed sample $\left(\phi_{\mathrm{RCP}}=0.65\right.$ for a polydispersity of $\left.\sim 3 \%\right)$ [16]. During slow sedimention, the particles start to order at the bottom wall of the sample container. No sticking to the glass wall was observed. Since the influence of the particle shape is most pronounced at highest compression, we analyzed the structures after sedimentation had fully completed. The particles were imaged using a Nikon TE 2000U inverted microscope equipped with a Nikon $\mathrm{C} 1$ confocal scanning laser head. Each frame contained more than 2000 particles. The variations between the frames were negligible and averaging only reduced the noise in the data. The enhanced ordering and layering of the structures at the wall-which does not persist in bulk - allowed a quantitative 2D analy-
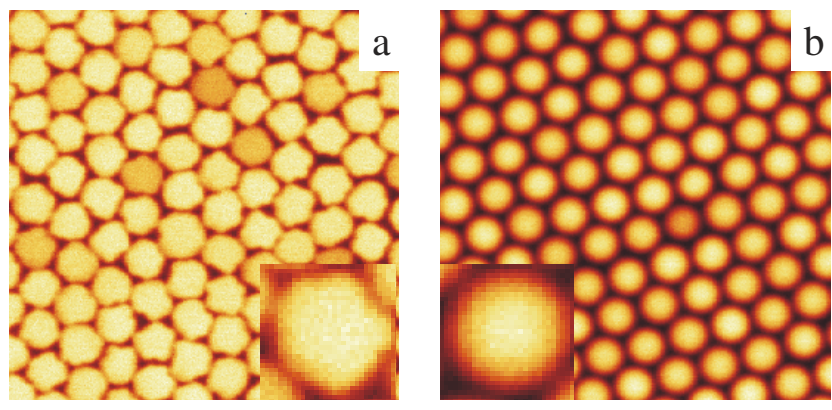

FIG. 1 (color online). Confocal microscopy images $(20 \times$ $20 \mu \mathrm{m}^{2}$ ) showing defect-free regions of packings of polyhedrals (a) and of spheres (b). The insets show the polyhedral and spherical shape of a single particle. 
sis of the structures present in the first layer of particles at the flat glass bottom wall of the container $[6,17]$. The centers of the particles were located using image-analysis software similar to that described in [18]. We verified that the polyhedral particle shape did not significantly affect the accuracy of the particle tracking. We focused on the structure of single-crystalline domains in the first layer at the wall, thereby leaving the influence of grain boundaries out of consideration. The surface fractions $\left(=N_{T} A_{p} / L^{2}\right.$, with $N_{T}$ the total number of particles in the image and $L^{2}$ the area of the image) were virtually equal: 0.846 and 0.852 , respectively, for the polyhedrals and the spheres.

Detailed real-space images of the structures of the spheres and the polyhedrals after complete sedimentation are presented in Fig. 1. First, we note that despite their shape the polyhedral colloids clearly exhibit hexagonal ordering. To quantify the structural differences observed in the first layer at the wall, we computed the radial distribution function $g(r)$ and the bond-orientational correlation function $g_{6}(r)[17,19]$, which is defined as

$$
g_{6}(r)=\left\langle\psi_{6}^{*}(0) \psi_{6}(r)\right\rangle
$$

with

$$
\psi_{6}\left(r_{i}\right)=\frac{1}{N} \sum_{j} \exp \left[6 i \theta\left(r_{i j}\right)\right]
$$

Here, $\psi_{6}$ is the local bond-orientational order parameter, where the summation $j$ runs over all, in total $N$, nearest neighbors of particle $i . \theta\left(r_{i j}\right)$ is the angle between the bond-vector connecting particles $i$ and $j$ and an arbitrary fixed reference axis. The \langle\rangle in Eq. (1) denote averaging over all pairs of particles and the index $i$ in Eq. (2) runs over all particles.

As shown in Fig. 2(a) the peaks in the $g(r)$ of the polyhedrals are markedly broadened and the structure in the $g(r)$ decays much faster in comparison to the $g(r)$ of the spheres. Moreover, just the second peak is weakly splitted, indicating local translational order only. The bondorientational correlation functions, which are shown in Fig. 2(b), exhibit clear differences as well. The decays of $g_{6}(r)$ indicate that the bond-orientational correlation in the polyhedral system is lost faster than in the crystal of the spheres. Also the local hexagonal order as illustrated by $\left\langle\left|\psi_{6}\right|\right\rangle$ (Table I), is smaller for the polyhedrals.

It is straightforward to see that a sphere with a randomly perturbed shape locally disturbs hexagonal packing. However, it is not directly clear how this noncommensurate shape affects the bond-orientational correlation at larger distances. To elucidate this, we studied the structure within one lattice orientation. We selected sets of particles along a line corresponding to one of the three lattice orientations, which we term a "lattice line." To decide if a particle belongs to a lattice line, we set a threshold of \pm 20 degrees for the maximum orientation difference between successive particles in a lattice line. Hence, we avoid kinks in the lattice lines due to defects $\left(>20^{\circ}\right)$ without neglecting the
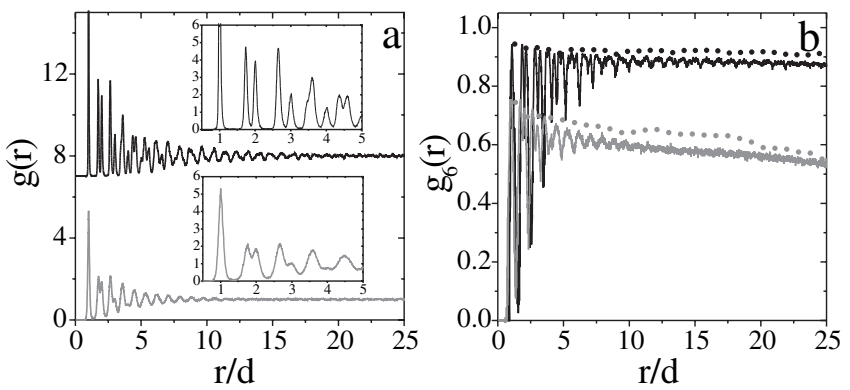

FIG. 2. (a) Pair correlation functions for the polyhedrals (gray) and the spheres (black). The latter has been vertically shifted for clarity. The insets show an enlargement of the first peaks to emphasize the differences between the polyhedrals and the spheres. (b) Bond-orientational correlation function for the polyhedrals (gray) and the spheres (black). The dotted curves are 1D bond-orientational correlation functions (as defined in the text), which are vertically shifted for an easy comparison to the decay of the corresponding 2D bond-orientational correlation function.

fluctuations $\left(<20^{\circ}\right)$. All the order parameters determined using these lattice lines were averaged over all three lattice orientations.

First, we calculated the mean orientation $\theta_{i}$ of every lattice line $i$ being the average over the orientations of all particles constituting lattice line $i$. The orientation of a particle $\theta$ is given by the average orientation of the bond vectors to its nearest neighbors in a lattice line. Subsequently, for all particles, the difference $\Delta \theta=\theta-$ $\theta_{i}$ was determined. The angle distribution $P(\Delta \theta)$ denotes the histogram of the whole set of $\Delta \theta$ 's. The angle distributions for both systems are distinctively different as shown in Fig. 3. The polyhedral distribution is much broader than the relatively sharp distribution of the spheres, pointing to significant bond-orientational fluctuations in the polyhedral lattice lines compared to those of the spheres. In both cases, the lattice lines are on average straight, which is illustrated by the Gaussian shapes of the angle distributions around zero. To probe how the bond-orientational fluctuations affect the bond-order correlation in a lattice line, we calculated the one-dimensional analog of Eq. (1): $g_{6}^{1 D}(r)=\left\langle\sum_{k} \sum_{l \neq k} \cos \left[6 \Delta \theta\left(r_{k l}\right)\right]\right\rangle$, where $\Delta \theta\left(r_{k l}\right)$ is the angle difference between the orientations of particles $k$ and $l$. The $g_{6}^{1 D}(r)$ for the spheres and the polyhedrals are shown as dotted lines in Fig. 2(b). It is obvious that the decay of $g_{6}^{1 D}(r)$ is completely similar to that of the envelope of $g_{6}(r)$. This directly confirms that the

TABLE I. Correlation lengths, power-law exponents, and local hexagonal order parameter for the single-crystalline domains of spheres and polyhedrals.

\begin{tabular}{lcc}
\hline \hline & Polyhedrals & Spheres \\
\hline Local hexagonal order parameter $\left\langle\left|\psi_{6}\right|\right\rangle$ & 0.85 & 0.95 \\
Translational correlation length $\xi_{T}(d)$ & 3.1 & 12.0 \\
Orientational correlation length $\xi_{O}(d)$ & 91 & 423 \\
Power-law exponent $\eta_{6}$ & 0.12 & 0.02 \\
\hline \hline
\end{tabular}




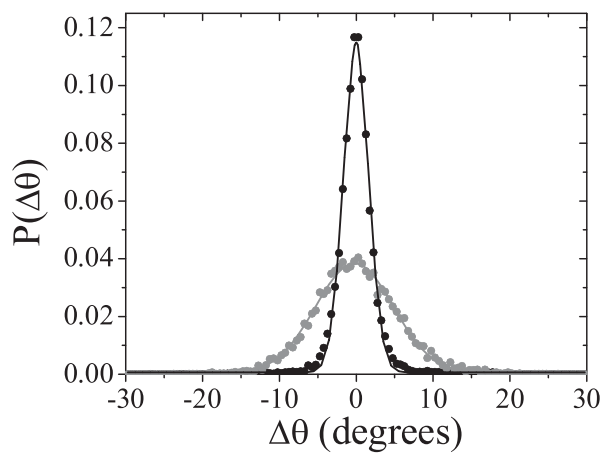

FIG. 3. The angle distribution from the lattice line analysis for the polyhedrals (gray) and the spheres (black). The solid lines are Gaussian fits to the data.

shape-induced bond-orientational fluctuations in the lattice lines disturb the bond-order correlations in the polyhedral structure.

To examine how these bond-orientational fluctuations affect the hexagonal structure, we compared the sets of particle positions for the polyhedrals and the spheres to perfect hexagonal grids with a lattice constant equal to the position of the first peak of $g(r)$. The bond order was aligned with the coordinate sets and the grid points were mapped onto the particle positions, obtaining a set of connected grid-coordinate pairs [20]. From these combinations, the root mean squared deviation $\Delta r=$ $\sqrt{\left\langle\vec{r}_{\text {coord }}-\left.\vec{r}_{\text {grid }}\right|^{2}\right\rangle}$ was calculated. Subsequently, minimizing $\Delta r$ by small rotations and translations resulted in the final mapping. We observe significant differences between the polyhedrals and the spheres in Figs. 4(a) and 4(d), which show the displacement vectors between the grid points and the corresponding particle positions [20]. First of all, the areas following the hexagonal grid are much smaller for the polyhedrals than for the spheres, even despite the bond-orientational single-crystalline nature of both systems. In other words, Fig. 4(a) suggests that the polyhedral structure consists of small translationally ordered domains separated by "mismatch lines" that percolate through the system. The completely randomized orientations of the displacement vectors for the polyhedrals [Fig. 4(b)] illustrate this "translational polycrystallinity," which directly points towards the absence of translational order. In contrast, the spheres only show some regions where all the particles exhibit similar small deviations from the hexagonal grid and no "mismatch lines." This is reflected by the anisotropic distribution of the orientations of the displacement vectors for the spheres [Fig. 4(e)].
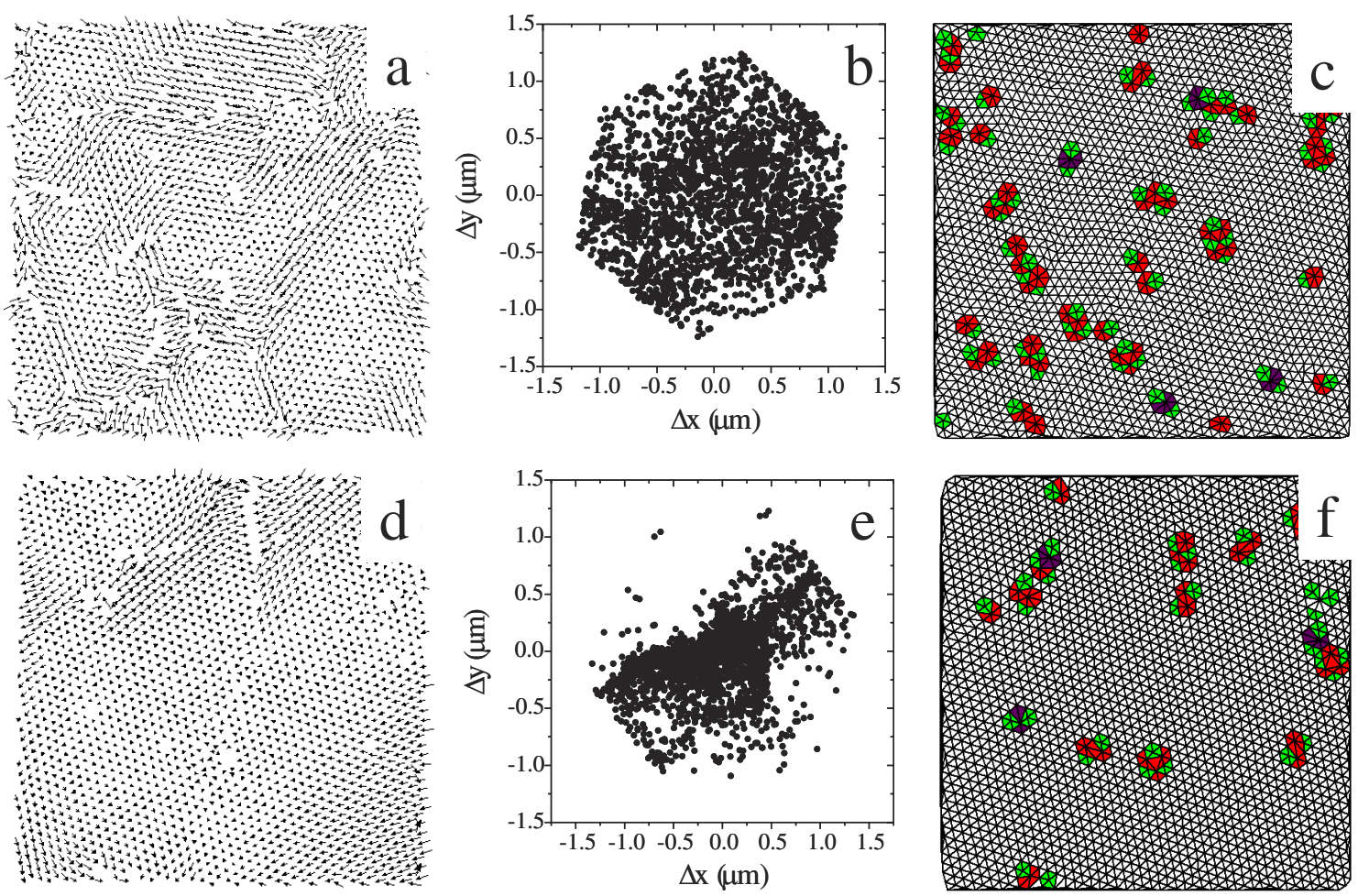

FIG. 4 (color online). Left column: Vector graphs $\left(100 \times 100 \mu \mathrm{m}^{2}\right)$ showing the displacement vectors for (a) the polyhedrals $(\Delta r=$ $0.31 d)$ and $(\mathrm{d})$ the spheres $(\Delta r=0.21 d)$. The length of the vectors has been increased by a factor of 2.5 for clarity. Central column: The $x$ and $y$ components of the displacement vectors connecting the grid points of a perfect hexagonal lattice to the particle positions for (b) the polyhedrals and (e) the spheres. Right column: Delaunay triangulations $\left(100 \times 100 \mu \mathrm{m}^{2}\right)$ identifying the defective particles by a color for (c) the polyhedrals and (f) the spheres. The color code is as follows: fourfold: blue, fivefold: green, sixfold: no color, sevenfold: red, and eightfold: purple. 
The location of the deviations from a hexagonal grid might be correlated to the presence of defects, which are identified using a Delaunay triangulation [see Figs. 4(c) and 4(f)]. Comparison with the vector graphs [Figs. 4(a) and 4(d)] reveals that some defects induce a small mismatch for the spheres. However, for the polyhedrals no obvious correlation is found. Although defects will cause mismatches, we argue that the bond-orientational fluctuations in the polyhedral system are predominantly responsible for the deviation from a hexagonal grid. Furthermore, we observe a different nature of the defects in both systems. In the sphere crystal, mainly dislocation pairs (typically vacancies) and hardly any free dislocations are observed, whereas in the polyhedral case much more free dislocations are identified.

The combination of the nature of the defects, the absence of translational order, and the presence of bondorientational order $\left[g_{6}(r)\right]$ point towards a hexatic nature of the polyhedral packing. To confirm this, we determined the translational correlation length $\xi_{T}$ [envelope of $g(r) \propto$ $\exp \left[\frac{-r}{\xi_{T}}\right]$, the orientational correlation length $\xi_{O}$ [envelope of $\left.g_{6}(r) \propto \exp \left[\frac{-r}{\xi_{0}}\right]\right]$, and the power-law exponent $\eta_{6}$ [envelope of $\left.g_{6}(r) \propto r^{-} \eta_{6}\right]$ for both systems (Table I). Applying the Kosterlitz-Thouless-Halperin-Nelson-Young formalism for two-dimensional melting [21-23], indeed confirms the crystalline nature of the spheres and the hexaticlike nature of the polyhedrals: the short range translational order is reflected by $\xi_{T}$ being only a few particle diameters, the (quasi) long-range orientational order by $\xi_{O}$ being more than 90 diameters and $\eta_{6}$ equaling 0.12 , which is clearly within the hexatic region $0<\eta_{6} \leq \frac{1}{4}$ [22].

Clearly the random polyhedral particle shape geometrically precludes the formation of a hexagonal crystal and leads to a hexaticlike structure. Apart from the nonsphericity, the random character of the polyhedral shape-the shape polydispersity - is a crucial property of the system. While identical hexamers for instance exhibit translational order at high densities [7,8], the translational order in our system is disturbed by the shape polydispersity geometrically frustrating hexagonal ordering. Similarly, it was reported that the inherent geometrical frustration for hexagonal ordering in two-dimensional arrays of bidisperse ball bearings, polydisperse hard spheres, and disks can suppress translational order, leading to a hexatic structure $[1,19,24]$ and even reentrant melting $[6,25]$.

We have demonstrated that next to size polydispersity, shape and shape polydispersity also significantly affect the ordering of particles. In particular, dense packings of hard spheres that have a small random perturbation in shape exhibit clear deviations from a perfect hexagonal lattice in terms of bond-orientational fluctuations. As a result, the translational order is short ranged while the bondorientational order is retained, resulting in a hexaticlike structure of the polyhedral colloids.

This work is part of the research programme of the Stichting voor Fundamenteel Onderzoek der Materie (FOM), financially supported by the Nederlandse Organisatie voor Wetenschappelijk Onderzoek (NWO). J.P.H. acknowledges support from the VW-stiftung.

*Electronic address: r.dullens@ physik.uni-stuttgart.de

[1] D. R. Nelson and M. Rubinstein, Philos. Mag. A 46, 105 (1982).

[2] P. G. Bolhuis and D. A. Kofke, Phys. Rev. E 54, 634 (1996).

[3] P. Bartlett and P. B. Warren, Phys. Rev. Lett. 82, 1979 (1999).

[4] S. Auer and D. Frenkel, Nature (London) 413, 711 (2001).

[5] P. Sollich, J. Phys. Condens. Matter 14, R79 (2002).

[6] R.P. A. Dullens and W. K. Kegel, Phys. Rev. Lett. 92, 195702 (2004).

[7] A.C. Branka, P. Pieranski, and K.W. Wojciechowski, J. Phys. Chem. Solids 43, 817 (1982).

[8] K. W. Wojciechowski, A. C. Branka, and M. Parrinello, Mol. Phys. 53, 1541 (1984).

[9] A. C. Branka and K. W. Wojciechowski, Mol. Phys. 72, 941 (1991).

[10] T. Schilling et al., Phys. Rev. E 71, 036138 (2005).

[11] N. R. Jana, Angew. Chem.. Int. Ed. Engl., Suppl. 43, 1536 (2004).

[12] A. Mehta, Granular Matter: An Interdisciplinary Approach (Springer-Verlag, New York, 1994).

[13] R.P. A. Dullens, E. M. Claesson, and W. K. Kegel, Langmuir 20, 658 (2004).

[14] G. Bosma et al., J. Colloid Interface Sci. 245, 292 (2002).

[15] E. H. A. de Hoog et al., Phys. Rev. E 64, 021407 (2001).

[16] W. Schaertl and H. Sillescu, J. Stat. Phys. 77, 1007 (1994).

[17] C. A. Murray, in Bond-orientational Order in Condensed Matter Systems, edited by K. Strandburg (Springer-Verlag, New York, 1992), pp. 137-215.

[18] J. C. Crocker and D. G. Grier, J. Colloid Interface Sci. 179, 298 (1996).

[19] D. R. Nelson, Defects and Geometry in Condensed Matter Physics (Cambridge University Press, Cambridge, 2002).

[20] J.P. Hoogenboom et al., Phys. Rev. E 69, 051602 (2004).

[21] J. M. Kosterlitz and D. J. Thouless, J. Phys. C 6, 1181 (1973).

[22] B. I. Halperin and D. R. Nelson, Phys. Rev. Lett. 41, 121 (1978).

[23] A. P. Young, Phys. Rev. B 19, 1855 (1979).

[24] A. V. Petukhov et al., Phys. Rev. Lett. 95, 077801 (2005).

[25] R. P. A. Dullens and W. K. Kegel, Phys. Rev. E 71, 011405 (2005). 